\title{
Cryptic Chromosome Rearrangements in Five Patients, with Normal and/or Abnormal Karyotypes, Associated with Mental Retardation, Autism and/or Epilepsy, Detected by BAC Genome Array-CGH
}

\section{Cabras V. ${ }^{3}$, Milia A. ${ }^{4}$, Montaldo C. ${ }^{2}$, Nucaro A. ${ }^{1}$}

${ }^{1}$ Genetic and Biomedical Research Institute - CNR, Cittadella Universitaria, Monserrato, Cagliari, Italy;

${ }^{2}$ Department of Surgical Sciences, Faculty of Medicine and Surgery, University of Cagliari, Cagliari, Italy;

${ }^{3}$ Department of Experimental Medical Pathology, Faculty of Medicine and Surgery, University of Cagliari, Cagliari, Italy;

${ }^{4}$ Department of Experimental Biology, Faculty of Medicine and Surgery, University of Cagliari, Cagliari, Italy

Received March 19, 2012; Accepted October 10, 2012.

Key words: Cryptic chromosome rearrangements - BAC genome array-CGH Genotype/phenotype correlation

\begin{abstract}
This report describes the usefulness of the BAC genome array-CGH platform in the detection of cryptic rearrangements. We examined ten patients with normal and/or abnormal karyotypes and dysmorphic features, associated with mental retardation, autism and/or epilepsy. This approach led us to discover further cryptic chromosomal rearrangements, not previously detected by conventional cytogenetic procedures, and allowed us to better delineate genotype/phenotype correlation. Our experience shows the validity of the BAC platform as a reliable method for genome-wide screening of chromosomal aberrations in patient with idiopathic mental retardation and/or in association with autism and epilepsy.
\end{abstract}

Mailing Address: Dr. Anna Lisa Nucaro, PhD., Genetic and Biomedical Research Institute - CNR, Cittadella Universitaria, ss 554, bivio Sestu, 09042 Monserrato, Cagliari, Italy; Phone: 39706754 654; e-mail: a.nuc@tiscali.it 


\section{Introduction}

We read with interest Zrnova et al's paper (2011) reporting the Czech Centre experience in the analysis of chromosomal aberrations in patients with mental retardation, using the oligo-array-CGH technique (CGH - comparative genomic hybridization). The use of genome array-CGH has recently dramatically improved the detection of cryptic chromosomal imbalances. This methodology utilizes mapped DNA sequences in a microarray platform for the detection of chromosomal deletions/duplications (Vermeesch et al., 2005; Edelmann and Hirschhorn, 2009; Sagoo et al., 2009).

We applied the BAC (bacterial artificial chromosome) genome array-CGH platform to study several patients with normal and/or abnormal chromosome karyotypes, previously investigated with conventional cytogenetic procedures, and found further cryptic rearrangements that had escaped traditional cytogenetic analysis, because of low power resolution. Our experience shows the validity of the BAC platform as a reliable method for genome-wide screening of chromosomal aberrations in patients with idiopathic mental retardation and/or in association with autism and epilepsy.

\section{Material and Methods}

Array comparative genomic hybridization

We applied a fast BAC genome array-CGH platform Cytochips Bluegnome, Technogenetics - Bouty (CytoChip BAC Summary Protocol Feb 2009, version 3) to study cryptic chromosomal rearrangements along the whole Genome. Cyto-Chips are high quality BAC microarrays (4898 BAC clones spotted in quadruplicate $0.6 \mathrm{Mb}$ ) and have been developed to screen the whole genome under high resolution in one hybridization. The metaphases are replaced by DNA clone fragments $(100-200 \mathrm{~kb})$ whose exact chromosomal location is known. This allows the detection of aberrations and makes it possible to map the changes directly onto the genomic sequence. Both DNAs are hybridized to cloned fragments (DNA) that are spotted on a glass slide (array). The DNA copy number aberrations are measured by detecting intensity differences. The principle is based on the co-hybridization of DNA sample (patient) and control (normal) which are labelled with two different fluorochromes ( $\mathrm{Cy} 3$ and $\mathrm{Cy} 5$ ) on the microarray. Double inverted labelling of sample and control is made to avoid false positivity. Images of the fluorescent signal are captured and analyzed. Red spots indicate loss of test DNA; green spots indicate gain of test DNA. The DNA copy number aberrations are subsequently measured by detecting intensity differences in the hybridization patterns of both DNAs.

In our study, we selected ten patients, 5 with normal karyotypes and 5 with abnormal karyotypes, associated with mental retardation, autism and/or epilepsy, to be revaluated by genome array-CGH. Informed written consent has been obtained from all the patients. 
FISH (fluorescence in situ hybridization) analysis was performed according to a standard protocol on metaphase slides that were prepared from lymphocyte cultures of peripheral blood. The slides were analyzed on a Diaplan Leica epi-fluorescence microscope and images were captured using a digital camera. FISH signals were examined both on metaphase chromosomes and interphase nuclei. BAC clones spotted on the array which showed gains or losses were subjected to FISH confirmation.

All the parents have been tested by FISH confirming of the novo origin of the rearrangements.

Briefly, we used $1 \mathrm{mg}$ of digested genomic DNA labelled with Cy5-dCTP or with Cy3 dCTP using random prime labelling (Bioprime Labeling Kit, Invitrogen). Labelled test and reference DNA were mixed and unincorporated nucleotides were removed using Autoseq G50 columns. After denaturation, the probe was applied to the array and hybridized overnight at $37{ }^{\circ} \mathrm{C}$ in a hybridization chamber. Slides were washed for 20 min $2 \times$ SSC $/ 0.05 \%$ Tween 20 at RT, 5 min $0.2 \times$ SSC at $60{ }^{\circ} \mathrm{C}, 5 \mathrm{~min} 1 \times \mathrm{SSC}$ at RT, $5 \mathrm{~min} 0.1 \times \mathrm{SSC}$ at $60^{\circ} \mathrm{C}$ and $5 \mathrm{~min} 0.1 \times \mathrm{SSC}$ at RT (RT - room temperature, SSC - sodium saline citrate). Arrays were scanned in a fluorescence confocal scanner (Spotter Arrayer mod. Affimetrix 417 and Scanner 428 array) and images were analyzed using specific software Bluefuse Cytochip (http://www.cytochip.com/productbluefuse).

Spots were defined by the software's automatic grid feature and manually adjusted whenever necessary. Spots showing no signal or defects were excluded from the data analysis, local background was subtracted, and the fluorescence intensity ratios of the two dyes, were calculated for each spot. The datasets were analyzed using Microsoft Excel. The mean ratios of four fluorescent signals (two signals from the duplicated clone on the array and two signals from the reverse experiment) for each clone were calculated. All analysis was done on log2 ratios. To reduce false positive results, clones showing a test/reference ratio value higher than 1 were considered gained and clones showing a test/reference ratio value lower than 1 were considered lost, but only if the results of all four fluorescent signals were consistent.

For this study, we have consulted the publicly accessible online database of Genomic Variants (http://projects.tcag.ca/variation), Ensemble (http://www.ensembl .org/index.html) and NCBI (http://www.ncbi.nlm.nih.gov/).

\section{Results}

We analyzed ten patients with normal and/or abnormal karyotypes, associated with congenital abnormalities and/or autism and epilepsy, by genome array-CGH, and found further cryptic rearrangements in 5 out of 10 (50\%).

Case 1: male, 9 years old. MR (mental retardation) associated with autism and epilepsy and dysmorphic features. Karyotype: 46,XY, inv dup del 8(p22;p23.1) 
(p23.2;pter). Array-CGH revealed a terminal loss in 8p23.2;pter, of about $4.66 \mathrm{Mb}$ (start 1846772- end 5459277). The duplication was detected by conventional QFQ (Q-fluorescence-Quinacrine) banding, at a resolution of about $10 \mathrm{Mb}$. In contrast, the deletion was not visible and was detected by genome array-CGH.

Different interesting genes map in the deleted region. In particular, we could indicate the CSMDI gene (Cub and sushi multiple Domains 1 ) as a good candidate for autism associated with mental retardation and epilepsy (Nucaro et al., 2011). Therefore, CSMDI is a giant gene, similar to CSMD3 (Cub and sushi multiple Domains 3) which maps in 8q, previously associated with autism (Floris et al., 2008) and epilepsy (Shimizu et al., 2003). It is possible to hypothesize that the haploinsufficiency of this gene could play an important role in this association.

Case 2: male, 11 years old. MR associated with epilepsy and dysmorphic features. Karyotype: 46,XY, r(14)(p13;q32)/46,XY,dup r(14). 48 out of 50 metaphases examined showed $r(14)$ and 2 a dup $r(14)$ (4\%). Array-CGH revealed loss of three BAC clones (BAC RPI I-4 I 7P24 at 14q32.33 (start 106196305- end 106364512) resulted as a polymorphic variant; BAC RPII-255G/2 in 14q22.1 (start 517905end 51944718); BAC RPII-I64G I 7 in 14q24.2 (start 71439536- end 71465495) in which we have found a candidate gene (SLC8A3, human gene for member 3 of solute carrier family) for epilepsy (Nucaro et al., 2010).

Case 3: female, 14 years old. MR associated with epilepsy. Karyotype: 46, XX, 146,XX, r(20)(p13;q13.3). 20 out of 50 metaphases examined showed the $r(20)$ (40\%). Array-CGH revealed a gain of two BACs, in 20 q11.21 (BAC RP4-733023 start 31,629,463- end 31,748,213) and BAC RP5-9/4B9 in 20q11.22 (start $33,103,098$ - end $33,224,086$ ) , along a region of about $1.5 \mathrm{Mb}$.

The BAC RP5-914B9 contains the DYNLRBI gene (Dynein, light chain, roadblocktype 1) (Jiang et al., 2001) a known protein coding, strongly expressed in the brain. No deletions were found at the site of ring formation nor alterations of two important genes responsible for epilepsy, CHRNA4 (neuronal acetylcholine receptor subunit alpha-4 precursor) and KCNQ2 (potassium voltage-gated channel subfamily KQT member 2), respectively, which map in the regions 20 q13.2-q13.33, about $1 \mathrm{Mb}$ to qter. We can also hypothesize that DYNLRB I could play a (silencing?) role in the function of these important genes.

Case 4: male, 16 years old. Severe MR associated with autism and epileptic encephalopathy. Karyotype: 46, XY. Array-CGH revealed a gain of a BAC in 17p11.2 (BAC RPI I-7807 start 19614001- end 19748680) of about $0.13 \mathrm{Mb}$, in the region of Potocki-Lupski Syndrome (Potocki et al., 2007).

Case 5: male, 5 years old. Severe MR associated with autism and epileptic encephalopathy. Karyotype: 46, XY. Array-CGH revealed a gain of a BAC in 17p11.2 
(BAC RPI I-7807 start 19614001- end 19748680) of about $0.13 \mathrm{Mb}$, in the PotockiLupski Syndrome region.

Cases 4 and 5 are not consanguineous. Cases 4 and 5 are enclosed in the DECIPHER database (https://decipher.sanger.ac.uk/patients) (Potocki-Lupski Syndrome, patients n. 256853 and 256873). The BAC RPI I-7807 contains three genes: ALDH3AI (aldehyde dehydrogenase dimeric NADP-preferring); ULK2 (serine/threonine-protein kinase); SLC47A2 (solute carrier family 47, member 2). SLC47A2 could be considered a good candidate gene for epilepsy. Figure 1 shows the array-CGH profiles of all the detected rearrangements. Table 1 shows the rearrangements detected in 5 patients by genome array-CGH. We interpreted aberrant signals from single clones as polymorphisms if the clones were listed in the publicly accessible online Database of Genomic Variants (http://projects.tcag.ca /variation).

\section{Discussion}

Genome-wide high resolution array-CGH was performed to investigate cryptic chromosome rearrangements in patients with normal and/or abnormal karyotypes associated with mental retardation, epilepsy and/or autism and we detected further cryptic chromosome imbalances in 5 out of 10 of the investigated cases which had been escaped cytogenetic standard procedures. Moreover, high resolution screening using array-CGH not only detects submicroscopic chromosomal imbalances, but also allows accurate delineation of the duplicated or deleted chromosomal segments. This is crucial for genotype-phenotype correlations and for identifying candidate genes involved in the development of mental disability, dysmorphic features, epilepsy and/or autism. The online databases to access phenotypes correlated to gene dose alterations, such as DECIPHER (http://www.sanger.ac.uk /PostGenomics/decipher/) or ECARUCA (http://www.ecaruca.net/), were helpful for interpreting the results of these analyses, excluding polymorphisms without clinical significance which are present in the population, including regions containing coding genes.

This technique allows us to identify SLC8A3 (human gene for member 3 of solute carrier family 8) as a candidate gene for epilepsy (Nucaro et al., 2010). It is a sodium-calcium exchanger electively expressed in the brain (Gabellini et al., 2002). The role of ionic channels is well demonstrated in epilepsy. These findings are very important to better genotype/phenotype correlation and to determine the clinical significance of these genomic imbalances.

In conclusion, this study provides evidence that the BAC genome array-CGH platform is an important and reliable tool, as well as oligonucleotide-based array-CGH, for detecting, mapping and characterizing cryptic chromosome rearrangements. Moreover, further studies are needed for a better genotype/ phenotype correlation. 

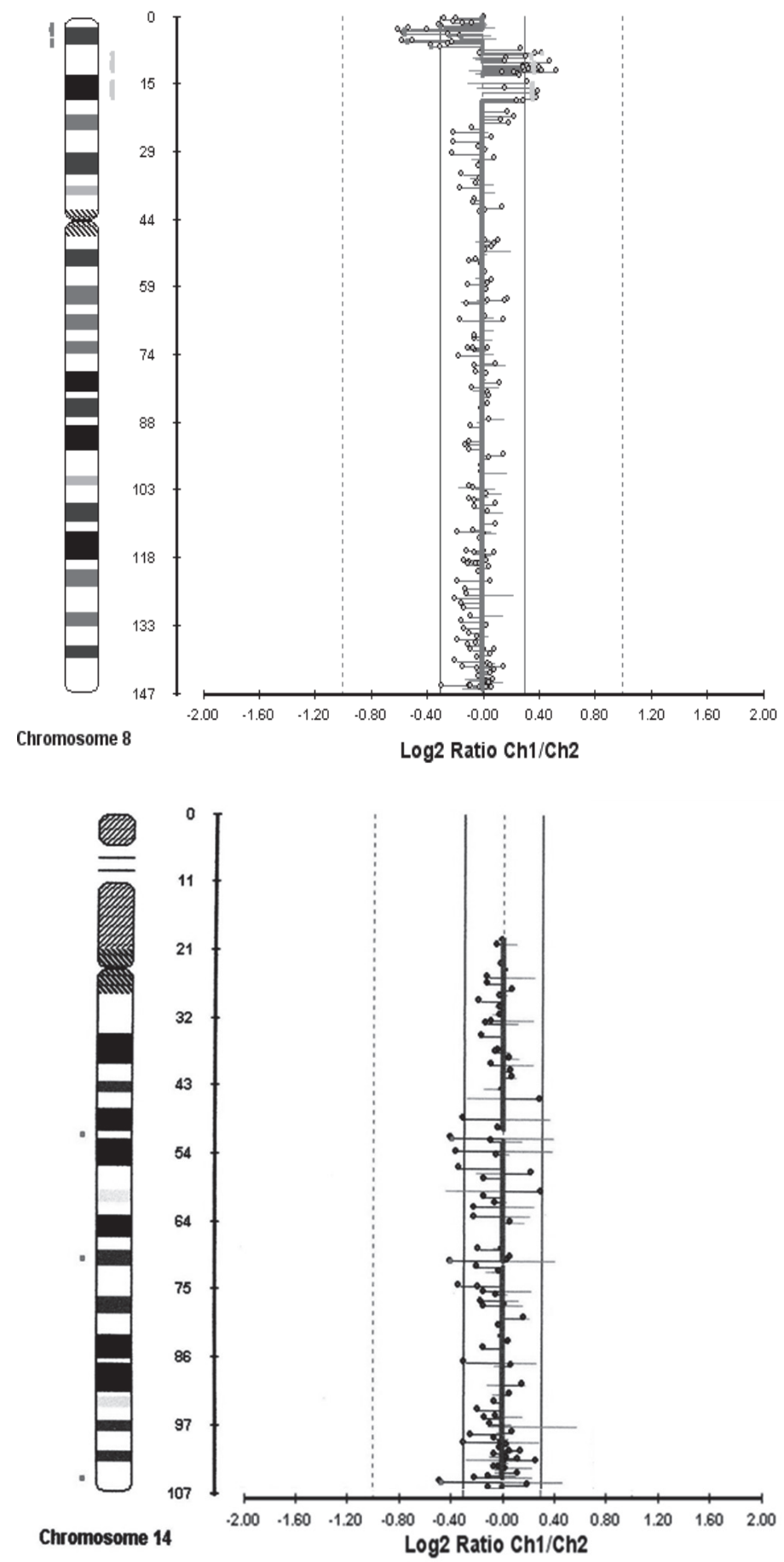


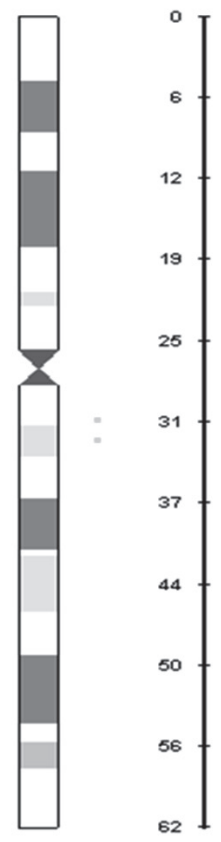

Chromosome 20

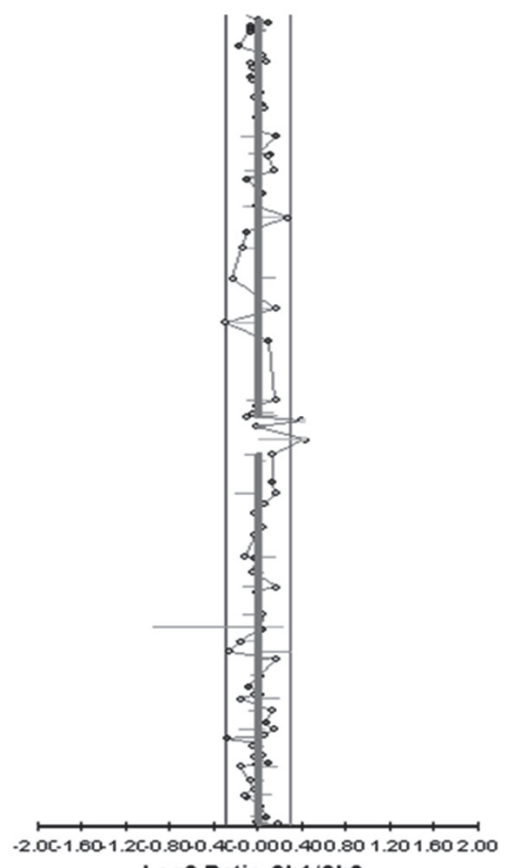

Log2 Ratio Ch1/Ch2

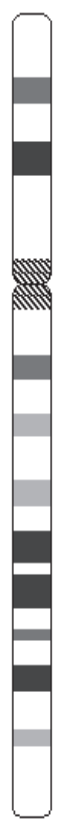

Chromosome 17

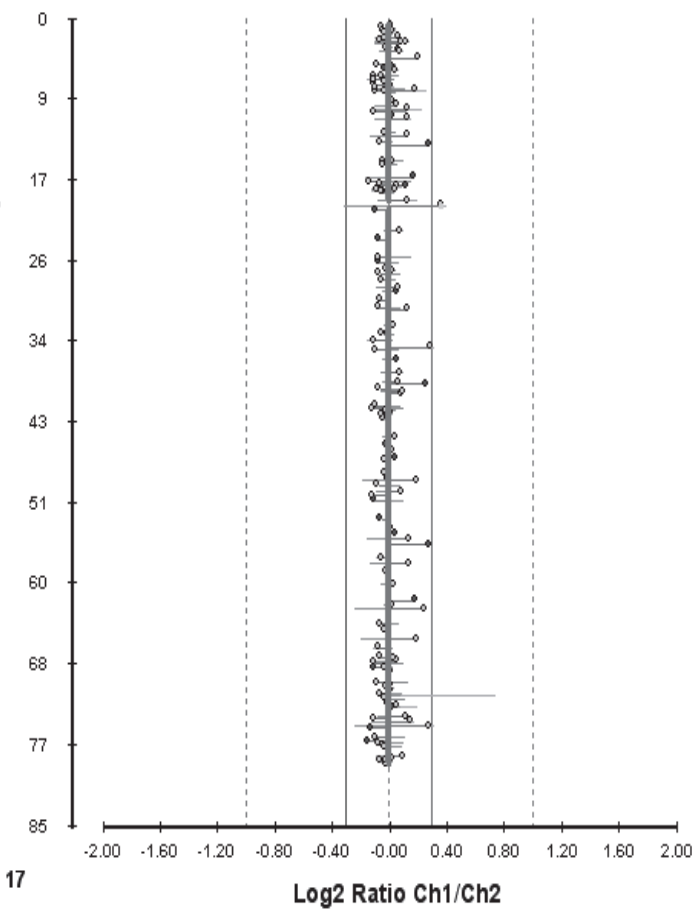

Cryptic Chromosome Rearrangements in Five Patients with Mental Retardation, Autism and Epilepsy 


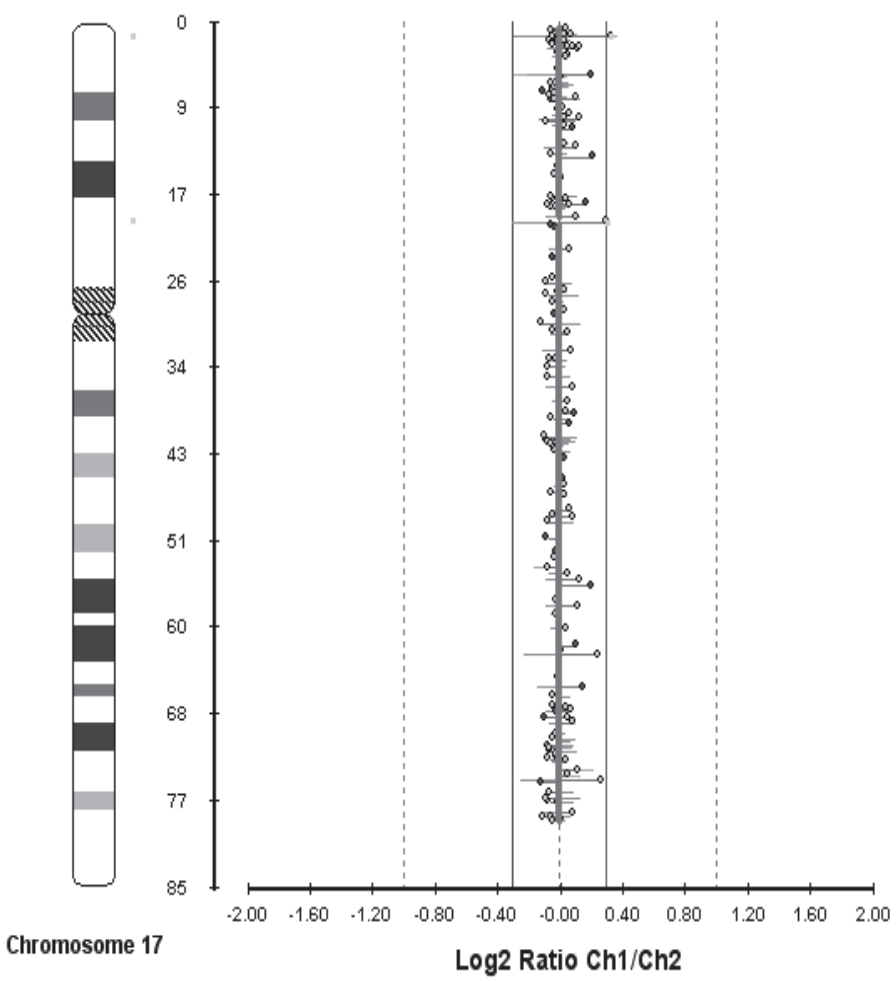

Figure 1 - CGH array profiles of the five detected rearrangements.

Acknowledgements: The authors thank to Prof. Carlo Cianchetti, Director of Pediatric Neurology Clinic, Azienda Ospedaliero-Universitaria, Cagliari, and the Clinicians from the Epilepsy Unit, for their collaboration in this study.

\section{References}

Edelmann, L., Hirschhorn, K. (2009) Clinical utility of array CGH for the detection of chromosomal imbalanced associated with mental retardation and multiple congenital anomalies. Ann. N. Y. Acad. Sci. 1151, 157-166.

Floris, C., Rassu, S., Boccone, L., Gasperini, D., Cao, A., Crisponi, L. (2008) Two patients with balanced translocations and autistic disorder: CSMD3 as a candidate gene for autism found in their common $8 \mathrm{q} 23$ breakpoint area. Eur. J. Hum. Genet. 16(6), 696-704.

Gabellini, N., Bortoluzzi, S., Danieli, G., Carafoli, E. (2002) The human SLC8A3 gene and the tissue-specific $\mathrm{Na}^{+} / \mathrm{Ca}^{2+}$ exchanger 3 isoforms. Gene 298,1-7.

Jiang, J., Yu, L., Huang, X., Chen, X., Li, D., Zhang, Y., Tang, L., Zhao, S. (2001) Identification of two novel human dynein light chain genes, DNLC2A and DNLC2B, and their expression changes in hepatocellular carcinoma tissues from 68 Chinese patients. Gene 281, 103-113.

Nucaro, A., Falchi, M., Pisano, T., Rossino, R., Boscarelli, F., Stoico, G., Milia, A., Montaldo, C., Cianchetti, C., Pruna, D. (2010) Ring chromosome 14 mosaicism:An unusual case associated with developmental delay and epilepsy, characterized by genome array-CGH. Am. J. Med. Genet. A 152, 234-236. 


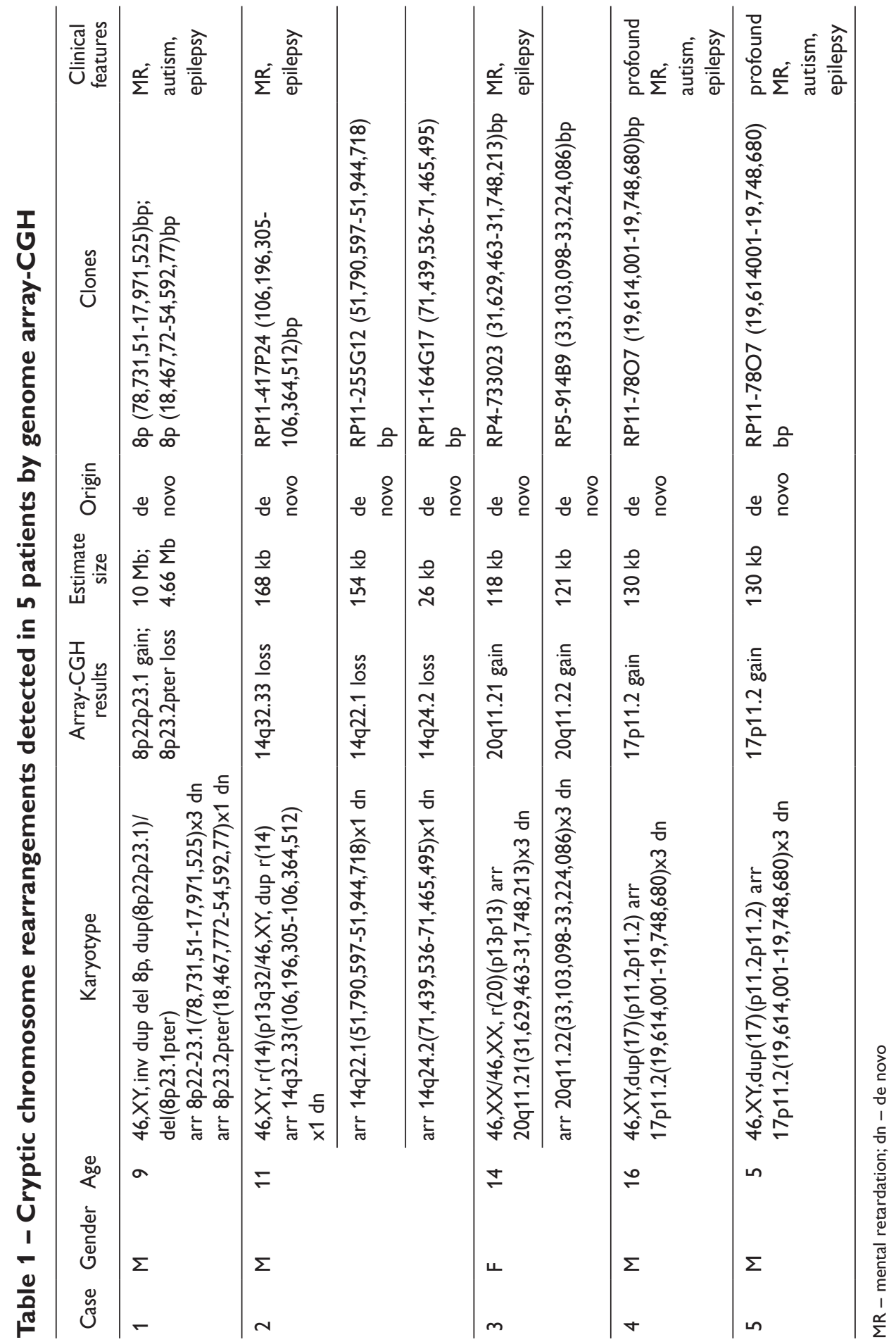


Nucaro, A., Pisano, T., Chillotti, I., Montaldo, C., Pruna, D. (2011) Chromosome 8p23.2-pter: a critical region for mental retardation, autism and epilepsy? Clin. Genet. 79(4), 394-395.

Potocki, L., Treadwell-Deering, P., Carvalho, C. M., Eifert, A., Friedman, E. M., Glaze, D., Krull, K., Lee, J. A., Lewis, R. A., Mendoza-Londono, R., Robbins-Furman, P., Shaw, C., Shi, X., Weissenberger, G., Withers, M., Yatsenko, S. A., Zackai, E. H., Stankiewicz, P., Lupski, J. R. (2007) Characterization of Potocki-Lupski syndrome (dup(17)(p11.2p11.2)) and delineation of a dosage-sensitive critical interval that can convey an autism phenotype. Am. J. Hum. Genet. 80(4), 633-649.

Sagoo, G. S., Butterworth, A. S., Sanderson, S., Shaw-Smith, C., Higgins, J. P., Burton, H. (2009) Array CGH in patients with learning disability (mental retardation) and congenital anomalies: updated systematic review and meta-analysis of 19 studies and 13,926 subjects. Genet. Med. 11(3), 139-146.

Shimizu, A., Asakawa, S., Sasaki, T., Yamazaki, S., Yamagata, H., Kudoh, J., Minoshima, S., Kondo, I., Shimizu, N. (2003) A novel giant gene CSMD3 encoding a protein with CUB and sushi multiple domains: a candidate gene for benign adult familial myoclonic epilepsy on human chromosome 8q23.3-q24.1. Biochem. Biophys. Res. Commun. 309, 143-154.

Vermeesch, J. R., Melotte, C., Froyen, G., Van Vooren, S., Dutta, B., Maas, N., Vermeulen, S., Menten, B., Speleman, F., De Moor, B., Van Hummelen, P., Marynen, P., Fryns, J. P., Devriendt, K. (2005) Molecular karyotyping: array CGH quality criteria for constitutional genetic diagnosis. J. Histochem. Cytochem. 53(3), 413-422.

Zrnova, E.,Vranova, V., Slamova, I., Gaillyova, R., Kuglik, P. (2011) Analysis of chromosomal aberrations in patients with mental retardation using the array-CGH technique: a single Czech Centre experience. Folia Biol. (Praha) 57, 206-215. 